 \\ Molecular Mechanisms of Nonphotochemical Quenching in the LHCSR3 Protein of Chlamydomonas reinhardtii
}

\author{
Gabriel de la Cruz Valbuena, ${ }^{\ddagger}, \#$ Franco V. A. Camargo, ${ }^{\ddagger}, \#$ Rocio Borrego-Varillas, ${ }^{\ddagger}, \#$ Federico Perozeni,

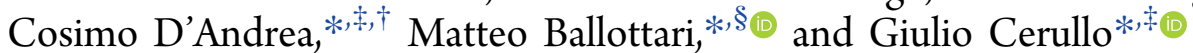 \\ ${ }^{\ddagger}$ IFN-CNR, Department of Physics, Politecnico di Milano, P.za L. da Vinci 32, 20133 Milano, Italy \\ ${ }^{\S}$ Dipartimento di Biotecnologie, Università di Verona, Strada Le Grazie 15, 37134 Verona, Italy \\ ${ }^{\dagger}$ Center for NanoScience and Technology@PoliMi, Istituto Italiano di Tecnologia, via Pascoli 70/3, 20133 Milano, Italy
}

Supporting Information

\begin{abstract}
Photosynthetic organisms possess photoprotection mechanisms from excess light conditions. The fastest response consists in the $\mathrm{pH}$-triggered activation of a dissipation channel of the energy absorbed by the chlorophylls into heat, called nonphotochemical quenching. In green algae, the pigment binding complex LHCSR3 acts both as a chlorophyll quencher and as a $\mathrm{pH}$ detector. In this work, we study the quenching of the LHCSR3 protein in vitro considering two different protein aggregation states and two $\mathrm{pH}$ conditions using a combination of

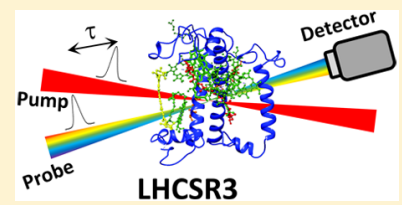
picosecond time-resolved photoluminescence and femtosecond transient absorption in the visible and NIR spectral regions. We find that the mechanisms at the basis of LHCSR3 quenching activity are always active, even at $\mathrm{pH} 7.5$ and low aggregation. However, quenching efficiency is strongly enhanced by $\mathrm{pH}$ and by aggregation conditions. In particular, we find that electron transfer from carotenoids to chlorophylls is enhanced at low $\mathrm{pH}$, while quenching mediated by protein-protein interactions is increased by going to a high aggregation state. We also observe a weak $\mathrm{pH}$-dependent energy transfer from the chlorophylls to the $S_{1}$ state of carotenoids.
\end{abstract}

$\mathrm{P}$ hotosynthetic organisms depend on the harvested sunlight to fuel their metabolic energy consumption. However, high-intensity illumination can be harmful to the organism. Under excess light conditions, the activity of the photosynthetic electron transfer chain saturates, inhibiting the photochemical relaxation of chlorophyll (Chl) singlet excited states $\left({ }^{1} \mathrm{Chl}^{*}\right)$. These states can then undergo intersystem crossing to the $\mathrm{Chl}$ excited triplet state $\left({ }^{3} \mathrm{Chl}^{*}\right)$, and the consequent formation of singlet oxygen $\left({ }^{1} \mathrm{O}_{2}{ }^{*}\right)$ causes photodamage or even cell death. ${ }^{1,2}$ Photosynthetic organisms evolved a variety of photoprotection mechanisms to prevent such harmful effects: differential gene expression and metabolic changes act on the long-term, ${ }^{2}$ while at the molecular level short-term mechanisms are rapidly activated upon increased irradiances. $^{3-5}$

One of these short-term responses is called nonphotochemical quenching (NPQ) and consists in the opening of channels for safe dissipation of the excitation energy as heat. NPQ consists of several processes, the fastest of which is called energy-dependent quenching $(\mathrm{qE}) .^{3-8}$ Several nonmutually exclusive molecular mechanisms for $\mathrm{qE}$ were proposed, all involving activation of a fast $(\sim p s)$ quenching process of ${ }^{1} \mathrm{Chl} *$. Strong electronic coupling of two adjacent Chls was suggested to lead to the formation of a charge transfer state that dissipates energy. ${ }^{9,10}$ All other $\mathrm{qE}$ mechanisms involve the interaction of Chls with carotenoids (Cars), which are suitable quencher molecules due to their low-energy short-lived dark states. ${ }^{11,12}$ One is energy transfer from ${ }^{1} \mathrm{Chl}^{*}$ to a lower energy excited dark state of the Car, such as $S_{1}{ }^{13-15}$ or the more recently proposed $S_{q}{ }^{16}$ with short $(\sim p s)$ lifetime; another is electron transfer from the Car to the photoexcited Chl, generating a Car radical cation and a Chl anion; ${ }^{17-20}$ a third mechanism is Car-Chl excitonic coupling, with the coupled state acting as excitation quencher. ${ }^{21}$ Often Cars quenching is related to the xanthophyll cycle (conversion of Car violaxanthin to zeaxanthin), which upregulates the NPQ process. $^{22-24}$

Previous studies demonstrated that $\mathrm{qE}$ is triggered by lumen acidification caused by saturation of the photosynthetic apparatus: Its onset thus requires both a sensor of the lumenal $\mathrm{pH}$ and a quencher for energy dissipation. In higher plants, the $\mathrm{pH}$ sensor is the nonpigment-binding protein PSBS, ${ }^{25,26}$ while other proteins from the light harvesting complex gene family are responsible for the energy dissipation process. ${ }^{9,13,19}$ In algae, pigment binding complexes named Light Harvesting Complex Stress Related (LHCSR) ${ }^{27}$ proteins are responsible for both $\mathrm{pH}$ detection and $\mathrm{Chl}$ quenching. In the model organism for green algae Chlamydomonas (C.) reinhardtii two LHCSR isoforms, LHCSR1 and LHCSR3, were observed to accumulate in the thylakoid membrane of the chloroplasts under excess illumination, with the latter being the main trigger for $\mathrm{qE}$ activation. ${ }^{27}$ LHCSR are trihelical proteins with Chl a, $\mathrm{Chl} \mathrm{b}$, and Car molecules bound to the helices and acid functional groups attached facing the lumenal part of the chloroplasts. $^{28-30}$ High light stress conditions generate a $\mathrm{pH}$

Received: April 25, 2019

Accepted: May 1, 2019

Published: May 1, 2019 

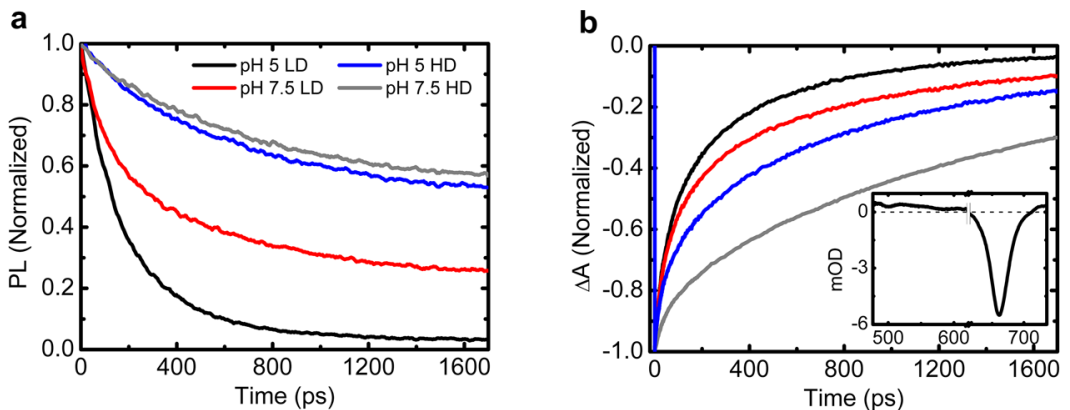

Figure 1. LHCSR3 kinetics traces measured by (a) TRPL (pump at $430 \mathrm{~nm}$, integrated from 650 to $760 \mathrm{~nm}$ ) and (b) TA at $680 \mathrm{~nm}$ (pump at 630 $\mathrm{nm})$. Inset: TA spectrum at $500 \mathrm{fs}$ ( $\mathrm{pH} 5 \mathrm{LD})$.

gradient across the thylakoid membrane, which is sensed by the acid groups of LHCSR3 and triggers its quenching activity. $^{29-32}$

The molecular quenching mechanisms in LHCSR proteins are still intensively studied and debated..$^{22,28,31}$ A zeaxanthindependent and a zeaxanthin-independent quenching process were identified in the LHCSR1 from the moss Physcomitrella (P.) patens, involving, respectively, energy transfer to the Car $\mathrm{S}_{1}$ state and formation of a Car radical cation. ${ }^{22}$ Both quenching mechanisms, energy transfer to Car $S_{1}$ and Car radical cation formation, have been also recently observed in vivo in the microalga Nannochloropsis oceanica strictly related to the presence of both zeaxanthin and the complex LHCX1, homologous to LHCSR subunits. ${ }^{33}$ Differently, in the case of C. reinhardtii, zeaxanthin has been demonstrated to be unnecessary for $\mathrm{qE}$ induction, ${ }^{30}$ opening the question about the quenching mechanisms underlying LHCSR3 activity in vivo. In this complex, a zeaxanthin-independent Car radical cation quenching mechanism was previously reported, being triggered at low $\mathrm{pH} .{ }^{30}$ However, this mechanism was measured in vitro, resulting in protein complexes with a fluorescence lifetime in the ns scale, incompatible with the strong quenching on tens to hundreds of ps time scales observed upon activation of LHCSR3-dependent quenching in vivo ${ }^{33,34}$ or in vitro in isolated PSII-LHCII-LHCSR supercomplexes. ${ }^{32}$ Recently, site directed mutagenesis ${ }^{31}$ allowed to obtain C. reinhardtii LHCSR3 variants with reduced quenching activity and $\mathrm{pH}$ inducibility in vivo. However, similar reduced quenching efficiency in vitro was obtained only when partial aggregation of the protein was induced by reducing the detergent concentration. $^{31}$ It is known that $\mathrm{qE}$ induction in vivo is accompanied by a change in protein-protein interactions, ${ }^{35}$ so these results suggest that protein-protein interactions induced in vitro by partial aggregation could simulate the quenching conditions occurring in vivo, even if the neighboring interacting proteins are not exactly the same in both cases. In this work, we employ ultrafast optical spectroscopy to understand the quenching mechanisms in LHCSR3 under different $\mathrm{pH}$ and aggregation conditions. We detect the onset of quenching pathways in LHCSR3 in vitro by changing the $\mathrm{pH}$ from 7.5 (neutral) to 5 (acid medium) and by reducing the concentration of detergent from high to low. We identify distinct $\mathrm{pH}$ - and aggregation-dependent mechanisms that are responsible for the $\mathrm{qE}$ activity of the protein.

LHCSR3 holoprotein was obtained upon pigment addition in an in vitro refolding process of recombinant apoprotein overexpressed in E. coli, as previously described. ${ }^{30}$ The refolded protein was characterized by an absorption spectrum with a peak in the Chl Qy region at $679.2 \mathrm{~nm}$ (Figure S1 in the
Supporting Information, SI) and fluorescence peaking at 683 $\mathrm{nm}$ (Figure S2), consistent with previous findings. ${ }^{30}$ Pigment binding properties of the refolded complex are reported in Table S1. To induce protein aggregation, the concentration of the detergent alpha-dodecyl maltoside $(\alpha$-DM) was decreased from $0.03 \%$ to $0.003 \%$ (see Materials and Methods in the SI). For simplicity, these conditions are referred to as high detergent (HD) and low detergent (LD) in the following. In principle, different $\mathrm{pH}$ conditions can induce different aggregation levels, so we tested the amount of aggregation using the fluorescence spectra at $77 \mathrm{~K}$. Figure S3 reveals the formation of oligomers characterized by increased far-red fluorescence only in the case of LD samples, with the strongest aggregate emission found in $\mathrm{pH} 5 \mathrm{LD}$. Hence, the $\mathrm{HD}$ samples act as controls for the effect of $\mathrm{pH}$ alone. We study the photophysics of the samples following photoexcitation of the Chls, combining time-resolved photoluminescence (TRPL), to determine the lifetime of ${ }^{1} \mathrm{Chl}^{*}$, and transient absorption (TA), to identify the formation and decay of dark excited states such as triplets, the Car $S_{1}$, and radical cation states.

Figure 1a shows the normalized TRPL decay kinetics integrated over the $650-760 \mathrm{~nm}$ spectral range under different $\mathrm{pH}$ and aggregation conditions. The $\mathrm{HD}$ sample at $\mathrm{pH} 5$ shows a faster decay compared to $\mathrm{pH} 7.5$, a trend that is also present under LD conditions with even shorter lifetimes (see Table S2 for time constants obtained by biexponential fits). This confirms that both aggregation and $\mathrm{pH}$ play a role in the overall quenching mechanism, with the detergent concentration being the most important factor, as the $\mathrm{pH} 7.5 \mathrm{LD}$ sample is more quenched than the $\mathrm{pH} 5 \mathrm{HD}$ one. Similar behavior was observed in the related protein LHCSR1 from $P$. patens. ${ }^{36}$ TRPL maps and spectra integrated over the entire temporal window of the experiment are reported in Figures S4 and $\mathrm{S} 5$, respectively.

Figure $1 \mathrm{~b}$ displays the TA kinetics at $680 \mathrm{~nm}$ of all samples (for TA maps and spectra in the visible, see Figures S6-S8) following excitation at $630 \mathrm{~nm}$. For all samples we used the same optical density and pump fluence, set at $6 \mu \mathrm{J} / \mathrm{cm}^{2}$ to minimize bimolecular annihilation effects ${ }^{37}$ (see Figure S9 for fluence-dependent dynamics of all quenched samples). At early times, the TA spectra of all samples consist of a negative peak around $680 \mathrm{~nm}$, due to ground state bleach (GSB) and stimulated emission (SE) of ${ }^{1} \mathrm{Chl}^{*}$ a, and a broad positive band from 470 to $600 \mathrm{~nm}$ corresponding to photoinduced absorption (PA) from ${ }^{1} \mathrm{Chl} *$. The GSB/SE dynamics follow the same trend observed in TRPL: shorter lifetimes at low $\mathrm{pH}$ and $\mathrm{LD}$, with the aggregation state prevailing. In the TA data a fast component is resolved in the $\mathrm{pH} 5 \mathrm{HD}$ and $\mathrm{LD}$ samples, whereas in TRPL measurements this difference is not observed 
due to the lower temporal resolution (20 ps as compared to $100 \mathrm{fs}$ ).

Most of the reported $\mathrm{qE}$ mechanisms involve interaction of the Chls with Cars. In our TA experiments, selective excitation of ${ }^{1} \mathrm{Chl}{ }^{*}$ ensures that any signal from the Car molecules must be due to an interaction with the photoexcited Chl. We thus focus on the 470-570 nm wavelength range (for TA maps and spectra, see Figures S7 and S8b) that contains the Car GSB (peaking at $500 \mathrm{~nm}$ ) and the Car triplet-triplet PA (peaking at $510 \mathrm{~nm}),{ }^{11,38}$ besides the previously mentioned broad PA of the ${ }^{1} \mathrm{Chl}^{*}$.

Figure 2a,b shows sequences of TA spectra for the most contrasting samples: $\mathrm{pH} 7.5 \mathrm{HD}$ and $\mathrm{pH} 5 \mathrm{LD}$, respectively. At
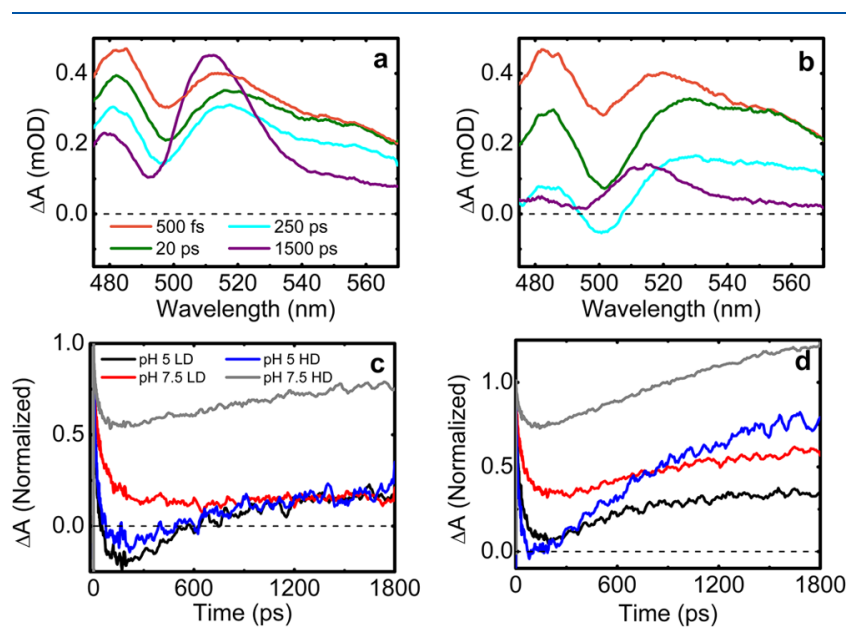

Figure 2. TA of LHCSR3 exciting ${ }^{1} \mathrm{Chl}^{*}$ at $630 \mathrm{~nm}$ for (a) $\mathrm{pH} 7.5$ $\mathrm{HD}$ and (b) pH $5 \mathrm{LD}$ conditions. Kinetics measured at (c) $500 \mathrm{~nm}$, showing the buildup of Car GSB, and (d) $510 \mathrm{~nm}$, showing the buildup of Car triplet-triplet PA.

early times, a negative peak overlapped in the broad PA spectrum of ${ }^{1} \mathrm{Chl} *$ is evident at $500 \mathrm{~nm}$ in both samples; as it matches the 0-0 peak of the $S_{0} \rightarrow S_{2}$ Cars transition, we assign it to Cars GSB. These results highlight coupling between Chls and Cars, previously observed ${ }^{21,22}$ and assigned to an ultrafast electrochromic shift of the carotenoid in response to the presence of the excited state of the Chl a. In Figures 2a,b we observe a buildup of the Cars GSB over tens of picoseconds, which is a trend we find on the other samples too (Figure 2c). This is more strongly present in the low $\mathrm{pH}$ samples, with the Car GSB becoming strong enough to prevail over the Chl PA and cause a change of sign of the overall TA signal. On the nanosecond time scale, we observe in Figure $2 \mathrm{~d}$ the formation of a PA band peaking at $510 \mathrm{~nm}$, attributed to the absorption of ${ }^{3} \mathrm{Car}^{*}{ }^{22}$ This state is populated via triplet-triplet energy transfer from the $\mathrm{Chl}$ to the Car, following intersystem crossing (ISC) in the Chl, according to the scheme ${ }^{1} \mathrm{Chl}^{*} \rightarrow{ }^{3} \mathrm{Chl}^{*} \rightarrow$ ${ }^{3} \mathrm{Car} *{ }^{36}$ Therefore, a longer lifetime of the ${ }^{1} \mathrm{Chl} *$ state corresponds to a higher efficiency for the ISC process and to a higher ${ }^{3} \mathrm{Car}^{*}$ signal. Consistently, the formation of the ${ }^{3} \mathrm{Car}^{*}$ PA band in Figure $2 \mathrm{~d}$ is more pronounced in the less quenched samples, with the highest triplet PA signal seen in $\mathrm{pH} 7.5 \mathrm{HD}$. The TA data in the Car region was adequately fitted with three time constants for all samples using a global analysis routine (see Figure S10 for the quality of the fits). ${ }^{39}$ The fit results are summarized in Table 1, and decay-associated spectra (DAS) of all samples are shown in Figure S11. We assign the calculated time constants to a fast multiexponential buildup of the
Table 1. Time Constants from the Global Fit Analysis of the TA Data from 480 to $570 \mathrm{~nm}$

\begin{tabular}{lccc} 
& $\tau_{1}(\mathrm{ps})$ & $\tau_{2}(\mathrm{ps})$ & $\tau_{3}(\mathrm{ps})$ \\
pH 7.5 HD & $13.4 \pm 0.1$ & $106 \pm 1$ & $1559 \pm 13$ \\
pH 5 HD & $10.3 \pm 0.1$ & $81 \pm 1$ & $718 \pm 6$ \\
pH 7.5 LD & $11.3 \pm 0.2$ & $101 \pm 1$ & $684 \pm 7$ \\
pH 5 LD & $9.0 \pm 0.1$ & $69 \pm 0.5$ & $477 \pm 2$ \\
\hline
\end{tabular}

carotenoid GSB ( $\left.\sim 10 \mathrm{ps}, \tau_{1} ; 70-100 \mathrm{ps}, \tau_{2}\right)$, followed by the rise of the triplet state in a longer time scale (480-1600 ps, $\left.\tau_{3}\right)$, which becomes faster in the more quenched samples due to the shorter lifetime of the ${ }^{1} \mathrm{Chl}^{*}$ state.

Comparison between TA spectra in Figures $1 \mathrm{~b}$ and $2 \mathrm{c}$ reveals a correlation between the quenching activity of LHCSR3 and the build-up Car GSB, pointing to the involvement of Cars in the $\mathrm{qE}$ process. However, this observation does not provide conclusive evidence of the quenching mechanism as we cannot distinguish if the process is associated with energy or charge transfer from Chls to Cars. Careful inspection of the TA dynamics at $560 \mathrm{~nm}$ for the $\mathrm{pH} 5$ samples (both $\mathrm{HD}$ and $\mathrm{LD}$ ), shown in Figure S12, reveals a weak increase of the PA signal. As this probe wavelength matches the peak of the characteristic intense $S_{1} \rightarrow S_{n}$ PA band of Cars, ${ }^{36}$ this demonstrates the presence of a weak quenching channel for ${ }^{1} \mathrm{Chl}^{*}$ via energy transfer to the Car $\mathrm{S}_{1}$. This channel is activated solely at low $\mathrm{pH}$ since it is not observed in the $\mathrm{pH} 7.5 \mathrm{HD}$ or LD samples.

Because Cars radical cations absorb in the NIR spectral range, following previous studies, ${ }^{22,30}$ we can directly assess the presence of electron transfer to the photoexcited Chls by extending our TA probe to the $850-1050 \mathrm{~nm}$ spectral range.

TA spectra at different pump-probe delays are reported in Figure 3a (see Figure S13 for the complete maps). At early times one observes a broad positive band, which is assigned to $\mathrm{PA}$ of the photoexcited $\mathrm{Chl}^{17}$ on a 20 ps time scale the signal shows a clear build-up, which is more pronounced at $880 \mathrm{~nm}$ with respect to $1050 \mathrm{~nm}$. Since the Chl population decays on this time scale (see Figure 1), the signal build-up must be associated with the formation of a new species. To estimate the spectrum of this species, we take the difference of the TA spectra recorded at 20 ps (when most of the build-up has occurred) and 500 fs. The resulting spectrum (inset of Figure 3a) peaks at $870 \mathrm{~nm}$, in excellent agreement with the PA spectrum of the lutein radical cation. ${ }^{40}$ Due to our limited spectral coverage, we cannot exclude partial involvement of the other Car present in LHCSR3, violaxanthin, for which the radical cation PA peaks at $830 \mathrm{~nm} .{ }^{40}$ The TA dynamics at 880 $\mathrm{nm}$, reported in Figure $3 \mathrm{~b}$, show that the Car radical cation is formed even at $\mathrm{pH} 7.5$, and in similar amounts regardless of the detergent concentration. At $\mathrm{pH} 5$ the formation of radical cation is enhanced, especially so for LD conditions. Global analysis of the NIR TA revealed three time constants for all samples (Table 2 and Figure S14), which were assigned to a fast multiexponential buildup of the Car radical cation $(\sim 10$ ps, $\left.\tau_{1} ; \sim 70 \mathrm{ps}, \tau_{2}\right)$ followed by a longer decay $\left(>400 \mathrm{ps}, \tau_{3}\right)$, which reflects both Car radical cation decay and Chl excited state decay. These observations correlate very well with the buildup of the Car GSB at $500 \mathrm{~nm}$ seen in Figure 2c. Therefore, we conclude that the buildup of the PA signal in the NIR and of the Cars GSB at $500 \mathrm{~nm}$ are predominantly associated with the formation of the Car radical cation, and that electron transfer from Car to $\mathrm{Chl}$ is an important 

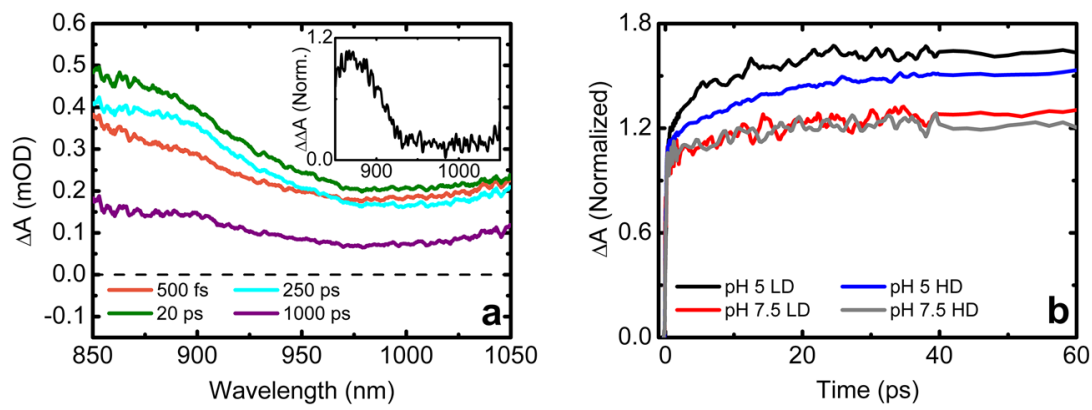

Figure 3. (a) NIR TA spectra of LHCSR3 for the pH5 LD sample following Chl excitation at $630 \mathrm{~nm}$. Inset: difference TA spectrum obtained by subtraction of the spectra at $20 \mathrm{ps}$ and $500 \mathrm{fs}$. (b) TA kinetics at $880 \mathrm{~nm}$ normalized by their average value over the first $300 \mathrm{fs}$ following excitation.

Table 2. LHCSR3 Time Constants Obtained by a Global Fit of the Data in the 880-1050 $\mathrm{nm}$ Spectral Range

\begin{tabular}{lrcc} 
& \multicolumn{1}{c}{$\tau_{1}(\mathrm{ps})$} & \multicolumn{1}{c}{$\tau_{2}(\mathrm{ps})$} & $\tau_{3}(\mathrm{ps})$ \\
pH 7.5 HD & $9.7 \pm 0.2$ & $74.7 \pm 3.1$ & $906.9 \pm 12.5$ \\
pH 5 HD & $10.1 \pm 0.1$ & $56.4 \pm 0.5$ & $772.9 \pm 2.9$ \\
pH 7.5 LD & $20.0 \pm 0.5$ & $91.3 \pm 4.4$ & $559.2 \pm 8.7$ \\
pH 5 LD & $10.2 \pm 0.1$ & $56.0 \pm 1.0$ & $409.7 \pm 1.1$ \\
\hline
\end{tabular}

quenching mechanism in LHCSR3 enhanced by $\mathrm{pH}$ and aggregation.

Our results demonstrate the $\mathrm{pH}$-dependent activation of electron transfer from Car to Chl in LHCSR3 and a concomitant weak excitation energy transfer to Car $S_{1}$. These findings can be compared to what has been recently reported in vivo in the case of LHCX1 from $N$. oceanica, ${ }^{33}$ where both quenching mechanisms were observed but only in the presence of zeaxanthin. Chl to Car energy transfer has been previously reported also in vitro for the LHCSR1 from $P$. patens, again only in the presence of zeaxanthin, ${ }^{22}$ or in the case of trimeric LHCII in aggregation conditions only. ${ }^{13}$ The case of LHCSR3 from $C$. reinhardtii herein reported is peculiar since Car radical cation formation and $\mathrm{Chl}$ to Car $\mathrm{S}_{1}$ energy transfer are both related to low $\mathrm{pH}$ and occur even in absence of zeaxanthin or aggregation. Electron transfer from Car to $\mathrm{Chl}$ is, however, further enhanced at $\mathrm{pH} 5$ and LD conditions, where LHCSR3 activity was previously correlated to its in vivo activity. ${ }^{31}$

The radical cation formation and the energy transfer to the Car $S_{1}$ are, however, insufficient to fully explain the quenching activity of LHCSR3. Indeed, these processes are, respectively, enhanced and activated at low $\mathrm{pH}$, while our data consistently show that the $\mathrm{pH} 7.5 \mathrm{LD}$ sample is more quenched than the $\mathrm{pH} 5 \mathrm{HD}$ one. Hence, there must be an additional quenching mechanism related to protein-protein interactions occurring in the LD samples, the condition where a closer correlation was found with the in vivo situation by analyzing the LHCSR3 activity in mutant variants. ${ }^{31}$ Aggregation-dependent quenching mechanisms have been previously reported both in vitro and in vivo in the case of different LHC complexes in higher plants, ${ }^{9,13,41,42}$ being revealed by the appearance of far-red fluorescence in quenched leaves. ${ }^{43}$ In the case of $C$. reinhardtii such far-red fluorescence was not detected in quenched cells. ${ }^{44}$ However, we note that upon NPQ induction a strong LHCSR3-dependent reduction of PSI fluorescence was also recently reported, ${ }^{45}$ likely masking the possible appearance of aggregation-dependent far-red fluorescence. Considering the substoichiometric content of LHCSR3 per PSII, ${ }^{46}$ LHCSR proteins upon protonation likely change their conformation inducing a different interaction with other LHC subunits rather than forming oligomers themselves.

In our LHCSR3 measurements, TRPL and TA data consistently show decay components for the ${ }^{1} \mathrm{Chl}^{*}$ signal with hundreds of ps time constant in LD conditions, with increased amplitude at low $\mathrm{pH}$ (see Figure 1), indicating the presence of a quenching mechanism related to aggregation and consequent protein-protein interactions. This is consistent with previous TRPL work on LHCSR1 from P. patens ${ }^{36}$ showing in LD conditions the presence of a 80 ps decay component with a red-shifted emission spectrum, which was attributed to the formation of aggregates. In the case of LHCII aggregates in vitro, besides energy transfer to Car S1, the main quenching mechanism proposed is the formation of a Chl-Chl charge transfer state, ${ }^{9}$ which could also be at the basis of the strong quenching observed in the case of LD samples here.

In conclusion, we presented a comprehensive characterization of the ultrafast photophysical mechanisms underlying $\mathrm{qE}$ in LHCSR3 using TRPL and TA in the visible and NIR regions with selective excitation of the Chls. The existence of signals attributable to the quenching species in all samples implies that the mechanisms of LHCSR3 quenching are always active, even at $\mathrm{pH} 7.5$ and low aggregation. However, quenching efficiency is strongly enhanced by the $\mathrm{pH}$ and by aggregation conditions. In particular, we find that both energy transfer to the $S_{1}$ of Car and electron transfer from Car to Chl are enhanced at low $\mathrm{pH}$, with the latter being further increased by aggregation, the condition where a strong correlation was found between in vitro LHCSR3 activity and in vivo NPQ induction. ${ }^{31}$ Since in vivo the lumen acidification is the trigger for LHCSR-dependent quenching in C. reinhardtii, it is important to note that protein-protein interactions are also tuned upon protonation of different thylakoidal proteins occurring at low $\mathrm{pH}$. In a final model we can thus propose that lumen acidification induces $\mathrm{qE}$ by switching LHCSR3 to a strongly quenched state correlated to increased charge transfer from carotenoids to chlorophylls and increased proteinprotein interactions. Quenching mechanisms in LHC proteins appear to be differently distributed among LHC subunits, with different quenching species being formed in different subunits and/or in different conditions. Possible quenching species were indeed observed in all the different LHC proteins investigated up to now, opening the unsolved question of how it is possible for these complexes with a high pigment density to maintain a stable and efficient light harvesting function rather than always causing excitation energy quenching. Considering the strong effect of the distance between Chl-Chl and/or Chl-Car for the formation of quenching species, fine-tuning of protein 
conformation and pigment organization is at the base of the light harvesting vs energy quenching activity of LHC subunits.

\section{ASSOCIATED CONTENT}

\section{S Supporting Information}

The Supporting Information is available free of charge on the ACS Publications website at DOI: 10.1021/acs.jpclett.9b01184.

Additional experimental data and global fits, materials and methods (PDF)

\section{AUTHOR INFORMATION}

\section{Corresponding Authors}

*E-mail: cosimo.dandrea@polimi.it.

*E-mail: matteo.ballottari@univr.it.

*E-mail: giulio.cerullo@polimi.it.

ORCID $\odot$

Matteo Ballottari: 0000-0001-8410-3397

Giulio Cerullo: 0000-0002-9534-2702

\section{Author Contributions}

\#These authors contributed equally.

\section{Notes}

The authors declare no competing financial interest.

\section{ACKNOWLEDGMENTS}

The research was supported by the ERC Starting Grant SOLENALGAE (679814).

\section{REFERENCES}

(1) Barber, J.; Andersson, B. Too much of a good thing: Light can be bad for photosynthesis. Trends Biochem. Sci. 1992, 17, 61-66.

(2) Takahashi, S.; Murata, N. How do environmental stresses accelerate photoinhibition? Trends Plant Sci. 2008, 13, 178-182.

(3) Matsubara, S.; Schneider, T.; Maurino, V. G. Dissecting longterm adjustments of photoprotective and photo-oxidative stress acclimation occurring in dynamic light environments. Front. Plant Sci. 2016, 7, 1690.

(4) Allahverdiyeva, Y.; Suorsa, M.; Tikkanen, M.; Aro, E. M. Photoprotection of photosystems in fluctuating light intensities. J. Exp. Bot. 2015, 66, 2427-2436.

(5) Ruban, A. V.; Johnson, M. P.; Duffy, C. D. The photoprotective molecular switch in the photosystem II antenna. Biochim. Biophys. Acta, Bioenerg. 2012, 1817, 167-181.

(6) Külheim, C.; Ågren, J.; Jansson, S. Rapid regulation of light harvesting and plant fitness in the field. Science 2002, 297, 91-93.

(7) Genty, B.; Briantais, J. M.; Baker, N. R. The relationship between the quantum yield of photosynthetic electron transport and quenching of chlorophyll fluorescence. Biochim. Biophys. Acta, Gen. Subj. 1989, 990, 87-92.

(8) Niyogi, K. K. Safety valves for photosynthesis. Curr. Opin. Plant Biol. 2000, 3, 455-460.

(9) Müller, M. G.; Lambrev, P.; Reus, M.; Wientjes, E.; Croce, R.; Holzwarth, A. R. Singlet energy dissipation in the photosystem II light-harvesting complex does not involve energy transfer to carotenoids. ChemPhysChem 2010, 11, 1289-1296.

(10) Wahadoszamen, M.; Margalit, I.; Ara, A. M.; van Grondelle, R.; Noy, D. The role of charge-transfer states in energy transfer and dissipation within natural and artificial bacteriochlorophyll proteins. Nat. Commun. 2014, 5, 5287.

(11) Polivka, T.; Frank, H. A. Molecular factors controlling photosynthetic light harvesting by carotenoids. Acc. Chem. Res. 2010, 43, 1125-1134.

(12) Frank, H. A.; Cua, A.; Chynwat, V.; Young, A.; Gosztola, D.; Wasielewski, M. R. Photophysics of the carotenoids associated with the xanthophyll cycle in photosynthesis. Photosynth. Res. 1994, 41, 389-395.

(13) Ruban, A. V.; Berera, R.; Ilioaia, C.; van Stokkum, I. H. M.; Kennis, J. T. M.; Pascal, A. A.; van Amerongen, H.; Robert, B.; Horton, P.; van Grondelle, R. Identification of a mechanism of photoprotective energy dissipation in higher plants. Nature 2007, 450, 575-578.

(14) Staleva, H.; Komenda, J.; Shukla, M.; Šlouf, V.; Kaňa, R.; Polívka, T.; Sobotka, R. Mechanism of photoprotection in the cyanobacterial ancestor of plant antenna proteins. Nat. Chem. Biol. 2015, 11, 287-291.

(15) Niedzwiedzki, D. M.; Tronina, T.; Liu, H.; Staleva, H.; Komenda, J.; Sobotka, R.; Blankenship, R. E.; Polívka, T. Carotenoidinduced non-photochemical quenching in the cyanobacterial chlorophyll synthase-HliC/D complex. Biochim. Biophys. Acta, Bioenerg. 2016, 1857, 1430-1439.

(16) Liguori, N.; Xu, P.; van Stokkum, I. H. M.; van Oort, B.; Lu, Y.; Karcher, D.; Bock, R.; Croce, R. Different carotenoid conformations have distinct functions in light-harvesting regulation in plants. Nat. Commun. 2017, 8, 1994.

(17) Holt, N. E.; Zigmantas, D.; Valkunas, L.; Li, X.; Niyogi, K. K.; Fleming, G. R. Carotenoid Cation Formation and the Regulation of Photosynthetic Light Harvesting. Science 2005, 307, 433-436.

(18) Avenson, T. J.; Ahn, T. K.; Zigmantas, D.; Niyogi, K. K.; Li, Z.; Ballottari, M.; Bassi, R.; Fleming, G. R. Zeaxanthin radical cation formation in minor light-harvesting complexes of higher plant antenna. J. Biol. Chem. 2008, 283, 3550-3558.

(19) Ahn, T. K.; Avenson, T.; Ballottari, M.; Cheng, Y. C.; Niyogi, K. K.; Bassi, R.; Fleming, G. R. Architecture of a charge-transfer state regulating light harvesting in a plant antenna protein. Science 2008, 320, 794-797.

(20) Li, Z.; Ahn, T. K.; Avenson, T. J.; Ballottari, M.; Cruze, J. A.; Kramer, D. A.; Bassi, R.; Fleming, G. R.; Keasling, J. D.; Niyogi, K. K. Lutein accumulation in the absence of zeaxanthin restores nonphotochemical quenching in the Arabidopsis thaliana npq1 mutant. Plant Cell 2009, 21, 1798-1812.

(21) Bode, S.; Quentmeier, C. C.; Liao, P.; Hafi, N.; Barros, T.; Wilk, L.; Bittner, F.; Walla, P. J. On the regulation of photosynthesis by excitonic interactions between carotenoids and chlorophylls. Proc. Natl. Acad. Sci. U. S. A. 2009, 106, 12311-12316.

(22) Pinnola, A.; Staleve-Musto, H.; Capaldi, S.; Ballottari, M.; Bassi, R; Polivka, T. Electron transfer between carotenoid and chlorophyll contributes to quenching in the LHCSR1 protein from Physcomitrella patens. Biochim. Biophys. Acta 2016, 1857, 1870-1878.

(23) Niedzwiedzki, D. M.; Sullivan, J. O.; Polivka, T.; Birge, R. R.; Frank, H. A. Femtosecond Time-Resolved Transient Absorption Spectroscopy of Xanthophylls. J. Phys. Chem. B 2006, 110, 2287222885.

(24) Arnoux, P.; Morosinotto, T.; Saga, G.; Bassi, R.; Pignol, D. A Structural Basis for the $\mathrm{pH}$-Dependent Xanthophyll Cycle in Arabidopsis thaliana. Plant Cell 2009, 21, 2036-2044.

(25) Li, X. P.; Bjorkman, O.; Shih, C.; Grossman, A. R.; Rosenquist, M.; Jansson, S.; Niyogi, K. K. A pigment-binding protein essential for regulation of photosynthetic light harvesting. Nature 2000, 403, 391395.

(26) Li, X. P.; Gilmore, A. M.; Caffarri, S.; Bassi, R.; Golan, T.; Kramer, D.; Niyogi, K. K. Regulation of photosynthetic light harvesting involves intrathylakoid lumen $\mathrm{pH}$ sensing by the PsbS protein. J. Biol. Chem. 2004, 279, 22866-22874.

(27) Peers, G.; Truong, T. B.; Ostendorf, E.; Busch, A.; Elrad, D.; Grossman, A. R.; Hippler, M.; Niyogi, K. K. An ancient lightharvesting protein is critical for the regulation of algal photosynthesis. Nature 2009, 462, 518-521.

(28) Liguori, N.; Novoderezhkin, V.; Roy, L. M.; van Grondelle, R.; Croce, R. Excitation dynamics and structural implication of the stressrelated complex LHCSR3 from the green alga Chlamydomonas reinhardtii. Biochim. Biophys. Acta, Bioenerg. 2016, 1857, 1514-1523.

(29) Mou, S.; Zhang, X.; Ye, N.; Dong, M.; Liang, C.; Liang, Q.; Miao, J.; $\mathrm{Xu}, \mathrm{D}$.; Zheng, Z. Cloning and expression analysis of two 
different LhcSR genes involved in stress adaptation in an Antarctic microalga, Chlamydomonas sp. ICE-L. Extremophiles 2012, 16, 193203.

(30) Bonente, G.; Ballottari, M.; Truong, T. B.; Morosinotto, T.; Ahn, T. K.; Fleming, G. R.; Niyogi, K. K.; Bassi, R. Analysis of LhcSR3, a protein essential for feedback de-excitation in the green alga Chlamydomonas reinhardtii. PLoS Biol. 2011, 9, e1000577.

(31) Ballottari, M.; Truong, T. B.; De Re, E.; Erickson, E.; Stella, G. R.; Fleming, G. R.; Bassi, R.; Niyogi, K. K. Identification of $\mathrm{pH}-$ sensing sites in the light harvesting complex stress-related 3 protein essential for triggering non-photochemical quenching in Chlamydomonas reinhardtii. J. Biol. Chem. 2016, 291, 7334-7346.

(32) Kim, E.; Akimoto, S.; Tokutsu, R.; Yokono, M.; Minagawa, J. Fluorescence lifetime analyses reveal how the high light-responsive protein LHCSR3 transforms PSII light-harvesting complexes into an energy-dissipative state. J. Biol. Chem. 2017, 292, 18951-18960.

(33) Park, S.; Steen, C. J.; Lyska, D.; Fischer, A. L.; Endelman, B.; Iwai, M.; Niyogi, K. K.; Fleming, G. R. Chlorophyll-carotenoid excitation energy transfer and charge transfer in Nannochloropsis oceanica for the regulation of photosynthesis. Proc. Natl. Acad. Sci. U. S. A. 2019, 116, 3385-3390.

(34) Amarnath, K.; Zaks, J.; Park, S. D.; Niyogi, K. K.; Fleming, G. R. Fluorescence lifetime snapshots reveal two rapidly reversible mechanisms of photoprotection in live cells of Chlamydomonas reinhardtii. Proc. Natl. Acad. Sci. U. S. A. 2012, 109, 8405-8410.

(35) Johnson, M. P.; Brain, A. P. R.; Ruban, A. V. Changes in thylakoid membrane thickness associated with the reorganization of photosystem II light harvesting complexes during photoprotective energy dissipation. Plant Signaling Behav. 2011, 6, 1386-1390.

(36) Pinnola, A.; Ballottari, M.; Bargigia, I.; Alcocer, M.; D’Andrea, C.; Cerullo, G.; Bassi, R. Functional modulation of LHCSR1 protein from Physcomitrella patens by zeaxanthin binding and low $\mathrm{pH}$. Sci. Rep. 2017, 7, 11158.

(37) van Oort, B.; Roy, L. M.; Xu, P.; Lu, Y.; Karcher, D.; Bock, R.; Croce, R. Revisiting the Role of Xanthophylls in Nonphotochemical Quenching. J. Phys. Chem. Lett. 2018, 9, 346-352.

(38) Polívka, T.; Sundström, V. Ultrafast Dynamics of Carotenoid Excited States-From Solution to Natural and Artificial Systems. Chem. Rev. 2004, 104, 2021-2072.

(39) Snellenburg, J. J.; Laptenok, S. P.; Seger, R.; Mullen, K. M.; van Stokkum, I. H. M. Glotaran: A Java-Based Graphical User Interface for the R Package TIMP. J. Stat. Software 2012, 49, 1-22.

(40) Amarie, S.; Standfuss, J.; Barros, T.; Kuhlbrandt, W.; Dreuw, A.; Wachtveitl, J. Carotenoid Radical Cations as a Probe for the Molecular Mechanism of Nonphotochemical Quenching in Oxygenic Photosynthesis. J. Phys. Chem. B 2007, 111, 3481-3487.

(41) Holleboom, C.; Gacek, D. A.; Liao, P.; Negretti, M.; Croce, R.; Walla, P. J. Carotenoid-chlorophyll coupling and fluorescence quenching in aggregated minor PSII proteins CP24 and CP29. Photosynth. Res. 2015, 124, 171-180.

(42) Ballottari, M.; Girardon, J.; Betterle, N.; Morosinotto, T.; Bassi, R. Identification of the Chromophores Involved in Aggregationdependent Energy Quenching of the Monomeric Photosystem II Antenna Protein Lhcb5. J. Biol. Chem. 2010, 285, 28309-28321.

(43) Miloslavina, Y.; Wehner, A.; Lambrev, P. H.; Wientjes, E.; Reus, M.; Garab, G.; Croce, R.; Holzwarth, A. R. Far-red fluorescence: A direct spectroscopic marker for LHCII oligomer formation in nonphotochemical quenching. FEBS Lett. 2008, 582, 3625-3631.

(44) Dinc, E.; Tian, L.; Roy, L. M.; Roth, R.; Goodenough, U.; Croce, R. LHCSR1 induces a fast and reversible pH-dependent fluorescence quenching in LHCII in Chlamydomonas reinhardtii cells. Proc. Natl. Acad. Sci. U. S. A. 2016, 113, 7673-7678.

(45) Girolomoni, L.; Cazzaniga, S.; Pinnola, A.; Perozeni, F.; Ballottari, M.; Bassi, R. LHCSR3 is a nonphotochemical quencher of both photosystems in Chlamydomonas reinhardtii. Proc. Natl. Acad. Sci. U. S. A. 2019, 116, 4212-4217.

(46) Tokutsu, R.; Minagawa, J. Energy-dissipative supercomplex of photosystem II associated with LHCSR3 in Chlamydomonas reinhardtii. Proc. Natl. Acad. Sci. U. S. A. 2013, 110, 10016-10021. 